\title{
Serum concentrations of CCL20/MIP-3alpha in women with endometriosis
}

\author{
Grzegorz Jabłoński ${ }^{1}$, Bogna Sobkiewicz ${ }^{1}$, Marcin Kaczmarek ${ }^{1}$, Adrian Krygier ${ }^{2}$, Jan Bieńkiewicz ${ }^{1}$, \\ Andrzej Malinowski ${ }^{1}$
}

${ }^{1}$ Department of Surgical, Endoscopic Gynecology and Oncology, Polish Mother's Memorial Hospital - Research Institute, Łodz, Poland ${ }^{2}$ Oxidative Stress Laboratory of the Center of Medical Laboratory Diagnostics and Screening, Polish Mother's Memorial Hospital Research Institute, Łodz, Poland

\begin{abstract}
Introduction: Endometriosis is a chronic disease defined by the presence of uterine mucosa outside the uterine cavity. Abnormal levels of cytokines, growth factors, adhesion molecules and metalloproteinases have been found in patients with endometriosis. A review of the literature revealed no papers on CCL20 serum levels in women with endometriosis.

Material and methods: The study included 32 women who underwent laparoscopy in the Polish Mother's Memorial Hospital-Research Institute, Lodz, Poland. Patients were divided into 2 groups: the study and control group. The study group was divided into three subgroups according to endometriosis form. Twenty patients were included in the study group and 12 patients acted as controls. CCL20 concentrations value were determined using a quantitative sandwich ELISA kit (R\&D Systems). Results were statistically analyzed by SPSS STATISTICS 24.0.0 software. A significance level of 0.05 was used.

Results: The mean serum level of CCL20 in the study group was $7.4 \mathrm{pg} / \mathrm{ml}$. In controls the mean value was $10.95 \mathrm{pg} / \mathrm{ml}$. The concentration of CCL20 was statistically significantly lower in the study group than in controls $(p=0.004)$. Within the study group the highest values were reported in patients with endometrial ovarian cysts $(8.55 \mathrm{pg} / \mathrm{ml})$, intermediate in the DIE subgroup $(8.24 \mathrm{pg} / \mathrm{ml})$ and the lowest in patients with peritoneal endometriosis $(6.74 \mathrm{pg} / \mathrm{ml})$. Differences between subgroups were not statistically significant $(p=0.385)$.

Conclusions: Our study revealed statistically significantly decreased CCL20 serum levels in women with endometriosis. No significant differences of CCL2O serum levels between patients with different forms of endometriosis were observed.
\end{abstract}

Key words: endometriosis, chemokines, cytokines.

\section{Introduction}

Endometriosis is a chronic disease defined by the presence of uterine mucosa (endometrium) outside the uterine cavity. These ectopic lesions contain both endometrial glands and stroma and can be found not only in the peritoneum and in ovaries forming superficial lesions or cysts (endometriomas) but can also infiltrate deep into underlying tissues (deep infiltrating endometriosis - DIE).

Etiopathogenesis of this condition still remains unclear. Although endometriosis affects $10-15 \%$ of women in reproductive age, up to $70 \%$ of female patients with chronic pelvic pain have been diagnosed with endometriosis and in women with infertility alone, the rate rises to $50 \%$. According to some reports, endometriosis mainly affects women aged 25-29, is more frequent in African Americans in comparison to Caucasians, but, interest- ingly, is less frequent in women declaring increased alcohol intake. Symptoms of endometriosis are diverse but usually involve: intermenstrual bleeding, painful periods (dysmenorrhea), painful sexual intercourses (dyspareunia), pain during micturition (dysuria) and defecation (dyschezia), and chronic pelvic pain (which often occurs periodically before each upcoming menses). However, the disease can also develop asymptomatically.

Endometriosis is one of the major causes of infertility and thus can result in a serious decline in quality of life [1-3]. An endometriosis classification system has been developed by the American Society of Reproductive Medicine. According to morphology and range of endometrial lesions, staging of endometriosis was divided into 4 types: minimal, mild, moderate and severe [4]. Endometriosis is also a chronic inflammatory and hormone-dependent disease. In particular, oestrogens 
have been considered to play a crucial role in its development, yet the stage of the disease itself also depends on various genetic, immunological, environmental and endocrine factors. Laparoscopy and subsequent histopathological assessment of specimens still remain the gold standard in the diagnosis of endometriosis. Regrettably, in many symptomatic women the proper diagnosis is often postponed for years, which is mainly attributed to the lack of reliable and dependable noninvasive diagnostic methods.

Several theories have been proposed to clarify the development of endometrial lesions. One of them explains it by the phenomenon of retrograde menstruation (Sampson's theory). According to it, during menstruation a part of desquamated endometrium gets into the peritoneal cavity via the Fallopian tubes and then undergoes typical hormonal activity transformations according to the cycle phase and, additionally, induces local inflammation [5]. However, retrograde menstruation - which can sometimes be observed during laparoscopy in the perimenstrual period-occurs in the majority of women (up to $90 \%$ ), but only a small share of them will develop true endometriosis. Cases of endometriosis were also reported in newborns, adolescent girls before menarche and in males, which suggests that other factors other than retrograde menstruation participate in its development [6].

According to another theory, some cells that line the visceral peritoneum are subject to metaplasia under the influence of environmental, inflammatory and hormonal factors. The explanation of this theory reaches back to early embryonal human development and is justified by common origins of peritoneum, pleura, paramesonephric (Müllerian) ducts and ovarian germinal epithelium: the coelom epithelium. Common embryonal origins of peritoneum and endometrium may explain how peritoneal cells can transform freely into endometrial tissue under the influence of some stimulating agents. Moreover, this theory clarifies the ability of endometrial lesions to develop in distant locations, which would be impossible to reach by retrograde menstruation (e.g. lungs) [6]. Recent literature data suggest a role of stem cells in pathogenesis of endometriosis through the ability of their relocation from the basal layer of the uterine mucosa into the peritoneal cavity also by means of retrograde menstruation. Proliferative and cellular differentiation capabilities then allow the stem cells to develop into endometrial implants [6].

Another theory claims that some immunological defects allow the development of endometrial lesions. Several findings seem to support this hypothesis. To begin with, the total macrophage count (which are a source of pro-inflammatory, chemotactic and angiogenic factors) is significantly elevated in peritoneal fluid in patients with endometriosis. Moreover, antiapoptotic agents themselves can be also identified both in the peritoneal fluid and plasma in these patients. Additionally, the occurrence of immature dendritic cells both in endometrial lesions and in peritoneum in women with endometriosis support the abovementioned hypothesis. It needs to be emphasized that these cells do not appear in the peritoneum in healthy women. The number of mature dendritic cells in both basal and functional layers of the endometrium in women with endometriosis is significantly lower than in those of healthy women. Moreover, some immune disorders may affect NK cell function. Interleukin 6 (IL-6) has already been described as a possible immunosuppressive agent that aims directly at NK cells and their cytostatic properties towards autologous endometrial cells. It has been proven that all the abovementioned immunological defects facilitate the development of endometrial lesions [6].

The interrelation between endometriosis and chemokines has lately been subject to scientific analysis. Abnormal levels of pro-inflammatory cytokines, growth factors, adhesion molecules, e.g. IL-1, IL-6, IL-8, IL-10, IL-13, IL-15, tumor necrosis factor $\alpha$ (TNF- $\alpha$ ), vascular endothelial growth factor (VEGF), monocyte chemoattractant protein-1 (MCP-1), soluble intercellular adhesion molecule-1 (sICAM-1) and metalloproteinases of extracellular matrix (MMP-2, MMP-3, MMP-7, MMP-9), have been found in the endometrium, plasma and also in the peritoneal fluid of patients with endometriosis [6-9]. These soluble agents exert an influence on both development and progression of endometriosis by stimulating cell growth, prompting angiogenesis and triggering endometrial stroma adhesion to the proteins of the extracellular matrix and also by suppressing apoptosis itself. These mechanisms allow for longer survival of endometrial cells in the peritoneal cavity and thus induce growth of endometrial lesions [3, 6-9].

In humans, chemokines are characterized by selective, chemotactic activity towards leukocytes and play a vital role in the process of leukocyte migration. Chemokines are small-cell cytokines (7-15 kDa), which act mostly as extracellular signaling molecules. Those agents mainly originate in lymphocytes, macrophages and monocytes. Both IL-1 and TNF- $\alpha$ stimulate synthesis and secretion of chemokines. Chemokines play a crucial role in the development of primary and secondary immune response, including molecular as well as humoral pathways.

According to the up-to-date literature, more than 50 chemokines and more than 20 chemokine receptors have been identified. Chemokines are divided into four main subfamilies: CXC, CC, CX3C and XC. Three groups (CC, CXC and CX3C) are particularly associated with the development of endometriosis [10-12]. The majority of chemokines are secreted during the inflammatory response, but some of them are produced in a constitutional manner in homeostatic conditions. 
Moreover, chemokines are involved in the processes of angiogenesis, autoimmunity, neoplasia and metastasis. The chemokine CCL20 (chemokine ligand 20) is named macrophage inflammatory protein (MIP)-3alpha, its activity is regulated by liver cells (liver activation regulated chemokine - LARC), and it belongs to the CC subfamily of chemokines, known as $\beta$-chemokines. The CCL20 gene locus is located on the long arm of chromosome 2 . In vitro it exhibits strong chemotactic activity towards immature dendritic cells, B lymphocytes and activated T lymphocytes T CD4+ and CD8+. CCL20 is able to trigger rapid adhesion of $\mathrm{T}$ lymphocytes to intercellular adhesion molecule (ICAM-1) and endothelial cells stimulated by TNF- $\alpha$, which suggests its essential role in the extravasation of lymphocytes. CCL20 shows a dual pattern of secretion: its production in the gastrointestinal (GI) tract is not very susceptible to regulation, whereas the liver, skin and lymphatic system secrete it in response to regulatory mechanisms. Depending on the conditions, it may have both homeostatic and pro-inflammatory function. Elevated concentrations of CCL20 were found in HIV-1 carriers, in nasopharyngeal cancer, pancreatic cancer and non-alcoholic fatty liver disease patients [1316]. The CCR6 receptor (CC chemokine receptor type 6) is the only known receptor for CCL20 and is expressed in the lymphatic system and also outside of it, including the spleen, lymph nodes, appendix, thymus, small intestine, pancreas, and peripheral blood leukocytes $-\mathrm{T}$ and B lymphocytes and dendritic cells [17-20].

In a recent systematic review on whether and how chemokines impact the etiopathogenesis of endometriosis 62 papers were investigated [21]. Twenty-seven various chemokines in peritoneal fluid, peripheral blood and eutopic endometrium were analyzed. Statistically significantly $(p<0.05)$ increased concentrations of chemokines were reported in $55 \%$ of analyzed papers. The most frequently investigated chemokines were CXCL8/IL-8 (45.1\% papers), CCL2/MCP-1 (37\%) and CCL5/RANTES (16.1\%). The paper concluded that CXCL8 seems to be the best marker for endometriosis. Its concentration in peritoneal fluid in women with endometriosis was significantly higher than in healthy controls in 15 out of 16 papers (93.75\%). However, only 6 out of 13 papers $(46,15 \%)$ revealed the increased plasma concentration of the abovementioned chemokine. Nevertheless, very few reports on how CCL20 impacts the development of endometrial lesions are available in current literature and the results still seem to be inconsistent. In infertile women with endometriosis no increased CCL20 concentration in peritoneal fluid was identified [22]. On the other hand, the CCL20 chemokine and its receptor CCR6 were found in endometrial ovarian cysts (endometriomas) [23]. Additionally, in the same paper, chemotaxis of Th17 cells induced by CCL20 was found. A review of current literature revealed no papers on the
CCL20 concentration in peripheral blood in women with endometriosis.

\section{Aim of the study}

The aim of this study was to assess the concentrations of CCL2O in patients with endometriosis and to attempt to determine the impact this chemokine has on the disease.

\section{Material and methods}

The study included 32 women of reproductive age who underwent laparoscopy in the Department of Surgical, Endoscopic Gynecology and Oncology, Polish Mother's Memorial Hospital Research Institute, Lodz, Poland in the timespan from June to September 2018. Patients were divided into 2 groups: study group - endometrial lesions were confirmed during laparoscopy; and control group - no evidence on endometrial lesion during laparoscopy. The study group was then divided into three subgroups according to endometriosis form confirmed during laparoscopy: peritoneal endometriosis, endometrial ovarian cysts (endometriomas) and deep infiltrating endometriosis (DIE). Twenty patients aged 23-47 (mean 33.45) were included in the study group and 12 patients aged 22 to 45 (mean 36.92) acted as controls. Within the study group peritoneal endometriosis was confirmed in 12 cases (60\%), endometrial ovarian cysts (endometriomas) in $4(20 \%)$ and DIE in 4 cases (20\%). The indications for laparoscopy were: confirmed endometriosis or suspicion of the disease - chronic pelvic pain or infertility. Every patient has provided written informed consent for participation in the study and agreed to have their blood sample collected before surgery. The protocol of the study has been approved by the Ethics Committee of Polish Mother's Memorial Hospital-Research Institute (Opinion No. 73/2017).

Blood samples were drawn from each patient before the surgery into a serum separator tube. After $30 \mathrm{~min}$ of incubation at room temperature the samples were centrifuged for $15 \mathrm{~min}$ at $2000 \times \mathrm{g}$. A part of sera was collected into a $1.5 \mathrm{ml}$ Eppendorf tube and frozen at $-80^{\circ} \mathrm{C}$. The human CCL20/MIP-3 $\alpha$ concentration values were determined using a quantitative sandwich ELISA kit for the quantitative determination of human CCL20 concentrations in cell culture supernates, serum and plasma (R\&D Systems, Inc. Minneapolis, MN 55413, USA). The values of CCL20 protein concentrations were determined according to the manufacturer's protocol. The standard curve concentrations used for the ELISA were $500 \mathrm{pg} / \mathrm{ml}, 250 \mathrm{pg} / \mathrm{ml}, 125 \mathrm{pg} / \mathrm{ml}, 62.5 \mathrm{pg} / \mathrm{ml}$, $31.2 \mathrm{pg} / \mathrm{ml}, 15.6 \mathrm{pg} / \mathrm{ml}$ and $7.8 \mathrm{pg} / \mathrm{ml}$. The detection range of this test was $7.8-500 \mathrm{pg} / \mathrm{ml}$. The minimum 
detectable dose was $0.47 \mathrm{pg} / \mathrm{ml}$. The standard curve was created using computer software (Gen5 Microplate Reader and Imager Software version 3.02, BioTek) able to generate a four-parameter logistic (4-PL) curve fit. The optical density of each well was evaluated using a microplate reader (Synergy $\mathrm{H} 1$ Microplate Reader, BioTek) set to $450 \mathrm{~nm}$ (with wavelength correction set to $540 \mathrm{~nm}$ ). Results were statistically analyzed by SPSS STATISTICS 24.0.0 software using $t$-test and ANOVA methods. A significance level of 0.05 was used.

\section{Results}

The mean serum concentration of CCL20/MIP-3 $\alpha$ in the study group was $7.4 \mathrm{pg} / \mathrm{ml}$ and the median was $8.12 \mathrm{pg} / \mathrm{ml}$. In controls the mean value was $10.9 \mathrm{pg} / \mathrm{ml}$,

Table 1. Serum level of CCL20/MIP-3 $\alpha$

\begin{tabular}{lcc}
\hline $\begin{array}{l}\text { Serum level of CCL20/ } \\
\text { MIP-3 } \alpha[\mathrm{pg} / \mathrm{ml}]\end{array}$ & $\begin{array}{c}\text { Study group } \\
(n=20)\end{array}$ & $\begin{array}{c}\text { Control group } \\
(n=12)\end{array}$ \\
\hline Average & 7.40 & 10.95 \\
\hline Median & 8.12 & 9.47 \\
\hline $\mathrm{Q}_{25}$ & 5.86 & 8.64 \\
\hline $\mathrm{Q}_{75}$ & 9.21 & 12.40 \\
\hline $\mathrm{SD}$ & 2.58 & 3.89 \\
\hline Min & 1.71 & 6.95 \\
\hline Max & 11.00 & 20.47 \\
\hline
\end{tabular}

median $9.47 \mathrm{pg} / \mathrm{ml}$ respectively (Table 1 ). The concentration of CCL20/MIP-3 $\alpha$ was statistically significantly lower in the study group than in controls ( $p=0.004$, Table 2, Fig. 1 ). Within the study group the highest values were observed in patients with endometrial ovarian cysts $(8.55 \mathrm{pg} / \mathrm{ml})$, intermediate in deep infiltrating endometriosis subgroup $(8.24 \mathrm{pg} / \mathrm{ml})$ and the lowest in patients with peritoneal form of the disease $(6.74 \mathrm{pg} / \mathrm{ml}$, Table 3). Differences within the study subgroups were not statistically significant $(p=0.385$, Table 4, Fig. 2).

\section{Discussion}

Our study is the first to reveal statistically significantly decreased serum levels of CCL20 in patients with endometriosis. Yet, it did not reveal any significant differences in the serum levels of this chemokine with regard to various forms of the disease (peritoneal endometriosis, ovarian cysts and DIE). Although the size of both study and control groups was rather limited (which is an obvious limitation of our study), our findings have clear statistical significance, which enables us to draw strong conclusions. Current literature lacks proper reports on serum CCL20 concentration and its impact on the etiopathogenesis of endometriosis, and at present $\mathrm{CXCL8/IL-8}$ seems to be the best serum marker for endometriosis. Current research efforts designed to extend our understanding of endometri-

Table 2. Comparison of CCL20/MIP-3 $\alpha$ serum levels between groups

\begin{tabular}{lcccccc}
\hline & \multicolumn{2}{c}{ Study group $(n=20)$} & \multicolumn{2}{c}{ Control group $(n=12)$} & \multicolumn{2}{c}{$t$-test } \\
\cline { 2 - 7 } & Average & SD & Average & SD & $t$ & $p$ \\
\hline CCL20/MIP-3 $\alpha$ serum level $[\mathrm{pg} / \mathrm{ml}]$ & 7.40 & 2.58 & 10.95 & 3.89 & -3.109 & 0.004 \\
\hline
\end{tabular}

Table 3. Serum levels of CCL20/MIP-3 $\alpha$ in endometriosis subgroups [pg/ml]

\begin{tabular}{lccc}
\hline $\begin{array}{l}\text { Serum levels } \\
\text { of CCL20/MIP-3 } \alpha\end{array}$ & $\begin{array}{c}\text { Peritoneal endometriosis } \\
(n=12)\end{array}$ & $\begin{array}{c}\text { Endometrial ovarian cyst } \\
(n=4)\end{array}$ & $\begin{array}{c}\text { Deep infiltrating endometriosis } \\
(n=4)\end{array}$ \\
\hline Average & 6.74 & 8.55 & 8.24 \\
\hline Median & 7.25 & 8.97 & 8.07 \\
\hline Q25 & 4.79 & 6.83 & 6.34 \\
\hline Q75 & 9.12 & 9.85 & 10.31 \\
\hline SD & 2.87 & 1.66 & 2.13 \\
\hline Min & 1.71 & 6.20 & 5.81 \\
\hline Max & 10.52 & 10.06 & 11.00 \\
\hline
\end{tabular}

Table 4. Comparison of CCL20/MIP-3 $\alpha$ serum levels between endometriosis subgroups

\begin{tabular}{lcccccccc}
\hline & \multicolumn{8}{c}{ Disease form } \\
\cline { 2 - 9 } & $\begin{array}{l}\text { Peritoneal endometriosis } \\
(n=12)\end{array}$ & $\begin{array}{c}\text { Endometrial ovarian cyst } \\
(n=4)\end{array}$ & $\begin{array}{c}\text { Deep infiltrating } \\
\text { endometriosis }\end{array}$ & ANOVA \\
\cline { 2 - 9 } & M & SD & M & SD & $(n=4)$ & SD & $F$ & $p$ \\
\hline $\begin{array}{l}\text { CCL20/MIP-3 } \alpha \\
\text { serum level }[\mathrm{pg} / \mathrm{ml}]\end{array}$ & 6.74 & 2.87 & 8.55 & 1.66 & 8.24 & 2.13 & 1.009 & 0.385 \\
\hline
\end{tabular}




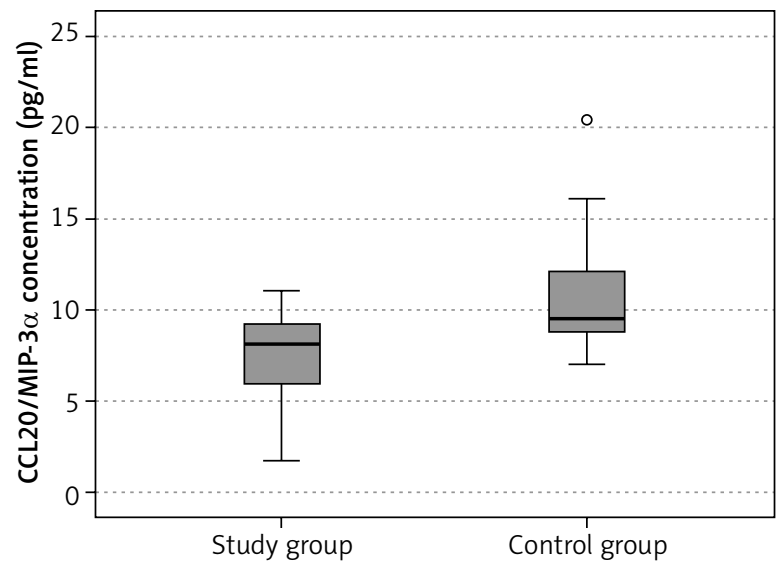

$$
\begin{aligned}
& \text { - Median } \\
& \square 25 \%-75 \% \\
& \text { I Min-Max }
\end{aligned}
$$

Fig. 1. Comparison of the serum levels of CCL20/MIP-3 $\alpha$ between groups

osis development focus on three major chemokines: CXCL8/IL-8, CCL2/MCP-1 and CCL5/RANTES [21]. ACcording to one paper, CCL20 levels in peritoneal fluid of infertile women with endometriosis were not altered [22]. Some reports show the CCL20 expression in both epithelial and stromal cells in endometrial lesions [23]. CCL20 has mainly chemotactic properties aimed at immature dendritic cells (DCs), B lymphocytes and activated T lymphocytes CD4+ and CD8+. Furthermore, CCL2O impacts on extravasation of lymphocytes and is secreted in humans both in constitutional and induced conditions and has both homeostatic and pro-inflammatory properties. Expression of cells which exhibit chemotaxis towards CCL2O was reported in endometrial lesions. There are some available reports on increased density of immature DCs (CD1a+) in the basal layer of endometrium in women with endometriosis. Furthermore, increased density of these cells was noted in peritoneal endometrial implants and peritoneum that was closely adjacent to them [24]. In another study higher expression of T lymphocytes CD4+, CD8+ and polyclonal activation of $B$ cells were detected in peritoneal fluid of women with endometriosis [25]. Also Th17 cell chemotaxis induced by CCL2O in women with endometrial ovarian cysts was reported [23].

The above mentioned analyses proving CCL20 presence in endometrial lesions and lack of its increased concentrations in peritoneal fluid and peripheral blood argue in favor of mainly local aggregation of CCL2O in endometrial implants. Reports on the role of immune cells with substantial chemotactic properties towards CCL2O in the progression of the disease could only further clarify this hypothesis.

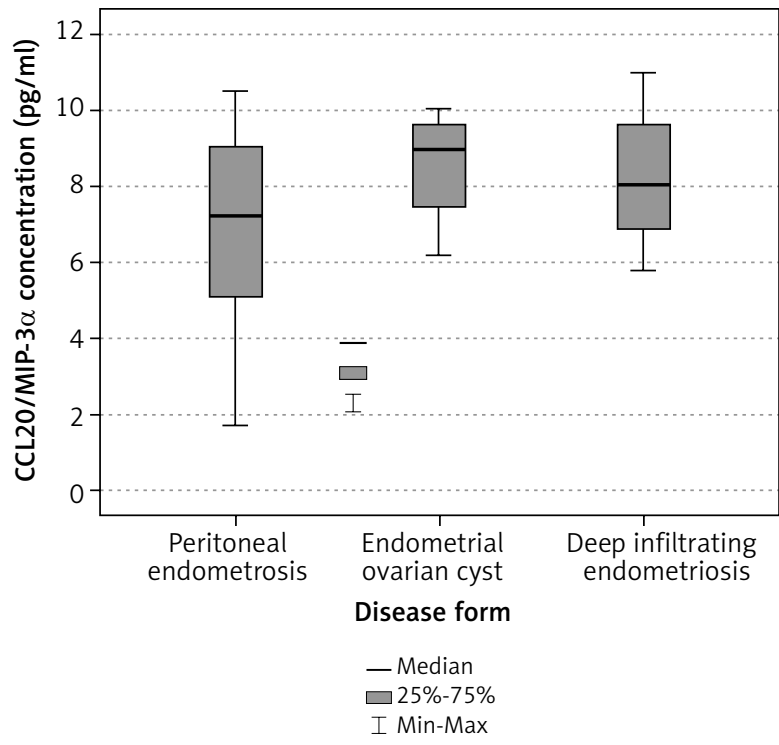

Fig. 2. Comparison of the serum levels of CCL20/MIP-3 $\alpha$ between endometriosis subgroups

In our study, conclusions need to be drawn carefully. Further studies are warranted in order to precisely investigate the role of CCL20 in the etiopathogenesis of endometriosis and clarify this matter, especially in larger groups.

\section{Conclusions}

Our study revealed statistically significantly decreased CCL20 serum levels in women with endometriosis. However, in this study no significant differences of serum CCL20 levels between patients with peritoneal endometriosis, endometrial ovarian cysts and deep-infiltrating endometriosis were observed.

\section{Disclosure}

The authors report no conflict of interest.

\section{References}

1. Parasar P, Ozcan P, Terry KL. Endometriosis: epidemiology, diagnosis and clinical management. Curr Obstet Gynecol Rep 2017; 6: 34-41.

2. Missmer SA, Hankinson SE, Spiegelman D, et al. Incidence of laparoscopically confirmed endometriosis by demographic, anthropometric, and lifestyle factors. Am J Epidemiol 2004; 160: 784-796.

3. Luckow Invitti A, Schor E, Martins Parreira R, et al. Inflammatory cytokine profile of cocultivated primary cells from the endometrium of women with and without endometriosis. Mol Med Rep 2018; 18: 12871296.

4. American Society for Reproductive Medicine. Revised American Society for Reproductive Medicine classification of endometriosis: 1996. Fertil Steril 1997; 67: 817-821.

5. Chen GY, Nuñez G. Sterile inflammation: sensing and reacting to damage. Nat Rev Immunol 2010; 10: 826-837.

6. Ahn SH, Monsanto SP, Miller C, et al. Pathophysiology and immune dysfunction in endometriosis. Biomed Res Int 2015; 2015: 795976. 
7. Agic $A, X u H$, Finas $D$, et al. Is endometriosis associated with systemic subclinical inflammation? Gynecol Obstet Invest 2006; 62: 139-147.

8. May KE, Villar J, Kirtley S, et al. Endometrial alterations in endometriosis: a systematic review of putative biomarkers. Hum Reprod Update 2011; 17: 637-653.

9. Chegini N, Roberts M, Ripps B. Differential expression of interleukins (IL)-13 and IL-15 in ectopic and eutopic endometrium of women with endometriosis and normal fertile women. Am J Reprod Immunol 2003; 49: 75-83.

10. Luster AD. Chemokines - chemotactic cytokines that mediate inflammation. N Engl J Med 1998; 338: 436-445.

11. Miller MD, Krangel MS. Biology and biochemistry of the chemokines: a family of chemotactic and inflammatory cytokines. Crit Rev Immunol 1992; 12: 17-46.

12. Nishida M, Nasu K, Narahara H. Role of chemokines in the pathogenesis of endometriosis. Front Biosci 2011; 3: 1196-1204.

13. Aziz N, Detels R, Chang LC, et al. Macrophage inflammatory protein3alpha (MIP-3 $\alpha$ )/CCL20 in HIV-1-infected individuals. J AIDS Clin Res 2016; 7: 587.

14. Chang KP, Hao SP, Chang JH. Macrophage inflammatory protein- $3 \alpha$ is a novel serum marker for nasopharyngeal carcinoma detection and prediction of treatment outcomes. Clin Cancer Res 2008; 14: 6979-6987.

15. Chu X, Jin Q, Chen H. CCL2O is up-regulated in non-alcoholic fatty liver disease fibrosis and is produced by hepatic stellate cells in response to fatty acid loading. J Transl Med 2018; 16: 108.

16. Vicari AP, Caux C. Chemokines in cancer. Cytokine Growth Factor Rev 2002; 13: 143-154.

17. Kohler RE, Caon AC, Willenborg DO, et al. A role for macrophage inflammatory protein-3 $\alpha / C C$ chemokine ligand 20 in immune priming during T cell-mediated inflammation of the central nervous system. J Immunol 2003; 170: 6298-6306.

18. Sikora J, Smycz-Kubańska M, Mielczarek-Palacz A, et al. Abnormal peritoneal regulation of chemokine activation: the role of IL-8 in pathogenesis of endometriosis. Am J Reprod Immunol 2017; 77: 12622.

19. Schutyser E, Struyf S, Van Damme J. The CC chemokine CCL20 and its receptor CCR6. Cytokine Growth Factor Rev 2003; 14: 409-426.

20. Hieshima K, Imai T, Opdenakker G, et al. Molecular cloning of a novel human CC chemokine liver and activation-regulated chemokine (LARC) expressed in liver. Chemotactic activity for lymphocytes and gene localization on chromosome 2. J Biol Chem 1997; 272: 5846-5853.

21. Borrelli GM, Abrão MS, Mechsner S. Can chemokines be used as biomarkers for endometriosis? A systematic review. Hum Reprod 2014; 29: 253-266.

22. Laudański P, Szamatowicz J, Oniszczuk M. Profiling of peritoneal fluid of women with endometriosis by chemokine protein array. Adv Med Sci 2006; 51: 148-152.

23. Hirata T, Osuga Y, Takamura M, et al. Recruitment of CCR6-expressing Th17 cells by CCL 20 secreted from IL-1 $1 \beta$-, TNF- $\alpha$-, and IL-17A-stimulated endometriotic stromal cells. Endocrinology 2010; 151: 5468-5476.

24. Schulke L, Berbic M, Manconi F, et al. Dendritic cell populations in the eutopic and ectopic endometrium of women with endometriosis. Hum Reprod 2009; 24: 1695-1703.

25. Riccio LDGC, Santulli P, Marcellin L, et al. Immunology of endometriosis. Best Pract Res Clin Obstet Gynaecol 2018; 50: 39-49 